Voix et Images

voixetimages

\title{
Les Oeuvres complètes de Nelligan : un diptyque désormais scriptible
}

\section{André Gervais}

Volume 18, numéro 1 (52), automne 1992

Les écritures masculines

URI : https://id.erudit.org/iderudit/201007ar

DOI : https://doi.org/10.7202/201007ar

Aller au sommaire du numéro

Éditeur(s)

Université du Québec à Montréal

ISSN

0318-9201 (imprimé)

1705-933X (numérique)

Découvrir la revue

Citer cet article

Gervais, A. (1992). Les Oeuvres complètes de Nelligan : un diptyque désormais scriptible. Voix et Images, 18(1), 156-162. https://doi.org/10.7202/201007ar d'utilisation que vous pouvez consulter en ligne.

https://apropos.erudit.org/fr/usagers/politique-dutilisation/ 


\title{
Les CEuvres complètes de Nelligan: un diptyque désormais scriptible
}

\author{
André Gervais, Université du Québec à Rimouski
}

C'est à l'occasion du cinquantième anniversaire de la mort d'Émile Nelligan, en novembre 1991, que parait l'importante édition, en deux tomes, des CEuvres complètes ${ }^{1}$, édition accompagnée de celle des $P o \dot{e}-$ mes autographes. Cet anniversaire est également souligné par un colloque $^{2}$ ainsi que par la publication de trois livres ${ }^{3}$.

Il y a matière, amplement. Mais, essentiellement, les CEuvres (enfin) complètes.

Je dis "enfin complètes * car y est rassemblé tout ce qu'on connaît maintenant, textuellement, de Nelligan ${ }^{4}$. L'édition critique, pour le tome I (Poésies complètes 1896-1941), est de Réjean Robidoux et Paul Wyczynski, pour le tome II (Poèmes et Textes d'asile 1900-1941) de Jacques Michon. Dans chaque tome, les textes proprement dits font entre 260 et 270 pages $^{5}$ (sur 646 pages et 615 pages respectivement), toutes les autres pages étant requises par le très élaboré et très utile développement de l'appareil critique: "Normes de l'édition", "Sigles, abréviations, signes conventionnels ", "Notes et variantes ", d'une part, "Avant-propos ", "Introduction ", * Chronologie ", " Bibliographie ", *Table alphabétique des titres et des incipit nelliganiens., etc., d'autre part (pour reprendre la table des matières du tome I).

Faut-il rappeler que Nelligan a été l'objet de la première édition critique jamais faite au Québec, préparée depuis 1947 par Luc Lacourcière et publiée en 1952 par Fides ${ }^{6}$ ? L'actuelle édition critique, qui relance la textualité de l'œuvre en perfectionnant l'appareil, tient compte, bien sûr, de tout ce qui s'est passé dans les études nelliganiennes?. 
Or, malgré tout, quelques questions, côté technique et côté discours sur, subsistent - que je poserai essentiellement à partir du tome I en ce qu'il reprend et réorganise ces Poéstes complètes 18961899, bien connues du grand public et des spécialistes.

1. Comment articuler les titres des deux tomes? D'une part les -poésies nelliganiennes proprement dites ", dites telles d'emblée (I, p. 10), d'autre part les * papiers de l'asile, souverainement aliénés, mais dotés néanmoins de leur signification particulière, dans la perspective du grand ensemble. (I, p. 10) ${ }^{8}$. Le "grand ensemble* en question est celui des CEuvres complètes, de l'« intégralité", de l'^entièreté ", de l'«authenticité *, de la - en un mot - "nelliganité - du "grand œuvre. (I, p. 10, 37 et 33). En fait, beaucoup plus du tome I que du tome II. Ne passe-t-il pas, entre "poésies" et "poèmes et textes", quelques appréciables connotations (approche plus traditionnelle/approche plus moderne, la poésie comme globalité/le poème et le texte comme unités indépendantes, etc.), et violemment, entre "complètes" et "d'asile ", la "ligne du partage des eaux. (I, p. 63), comme si les EEuvres ne pouvaient être vraiment complètes sans le tome II dont la matière, pour une bonne part, est pourtant intégrée aux - avalées, dirait Réjean Ducharme, par les - notes du tome I.

2. Dans leur introduction respective, les trois éditeurs sont loin d'avoir le même avis sur la matière de ce tome II. Robidoux et Wyczynski écrivent:

Le constat rétrospectif que le lecteur posthume en tire montre une œuvre complète doublement pathétique, à la fois parfaite et défaite. L'œuure dure donc et continue jusqu'à 1941, soumise seulement à la plus implacable entropie. (I, p. 26)

Souvent occupés à dire et redire leur difficulté à accepter ce corpus non légitimé, ils dénient toute capacité au Nelligan qui, à l'asile, •perd tout contrôle sur sa création. (I, p. 20), aucun des poèmes et textes qu'il écrit - écrit ou transcrit, (re)monte ou démonte, signe autrement, etc.: maghane, dirait encore Ducharme - ne mettant "à proprement parler en branle les facultés créatrices. (I, p. 26): : toutes choses sans réelle invention. (I, p. 74). Parallèlement, Michon parle du *laboratoire complexe de la création poétique. (II, p. 9) de Nelligan qui, à l'asile, accumule non seulement fautes et erreurs diverses qu'une recherche à la fois simple (orthographe, grammaire) et complexe (avant-texte) permet de constater et d'apprécier, mais aussi constructions et jeux divers " révélateurs des préoccupations du poète, de l'évolution de son œuvre et de son travail sur les textes. (II, p. 19). Il n'y a pas de doute: le même objet n'est pas le même objet ${ }^{9}$. 
3. Pourtant, et bien qu'à l'asile Nelligan -tente de reproduire tant bien que mal ses anciens poèmes avec les moyens d'une intelligence affaiblie. (I, p. 41), Robidoux et Wyczynski estiment que les leçons de plusieurs de ces reproductions sont quand même plus : lucides * que celles de l'édition princeps (Dantin, 1903-1904) ou de la précédente édition critique (Lacourcière, 1952), et fondent sur elles une part de la nelliganité du tome I: “C'était un grand vaisseau.. [...]* au lieu de *Ce fut un grand vaisseau [...]. (.Le Vaisseau d'Or •, v. 1; I, p. 312), « Et dans l'Éden d'or [...]* au lieu de «Et dans l'Éden [...]* («Fantaisie créole", v. 14; I, p. 217), par exemple. Beau paradoxe! Les "leçons", terme technique, n'est-ce pas aussi un lapsus?

\section{Robidoux et Wyczynski estiment à juste titre qu'une}

comparaison serrée des textes de Nelligan parus entre 1898 et 1904 permet de constater au surplus que Dantin, sans l'avouer explicitement, a fait bon nombre de changements (ponctuation, mots, expressions, prosodie). (I, p. 32-33)

Par ailleurs, dans un exemplaire de l'édition princeps, Dantin, en 1909, a fait d'autres corrections. Que penser de celle-ci, par exemple? Dans * Les Corbeaux * (I, p. 273), "nos ténèbres " (v. 7) devient "mes vertèbres . D'une part, cette double correction (ténèbres/vertèbres, nos/mes) ressemble, à s'y méprendre, à une réecriture. Comment savoir ici si Dantin a corrigé selon les manuscrits qu'il aurait gardés ou dont il se souvenait - on le voit, cela commence déjà à ressembler à Nelligan luimême retravaillant, à l'asile, ses poèmes... - ou, par un psychologisme déjà bien installé, selon ce qu'il pensait être plus conforme à l'idée qu'il se faisait ou qu'il aurait aimé qu'on se fasse de la poésie de Nelligan. D'autre part, le fait que dans les après-textes on retrouve et "mes vertèbres" (II, p. 132) et "nos ténèbres* (II, p. 289) dit peut-être qu'il y a, dans le cas de ce poème, quelque chose d'indécidable, quelque chose comme * c'est le témoignage de la victime contre celui de l'agresseur *

Sans être en mesure, semble-t-il, de lire dans le tome I le texte de Nelligan, nous lisons - et lirons - le texte nelliganien, c'est-à-dire - l'état normal (régulier et policé) recherché par le Nelligan d'avant l'internement ${ }^{10}$. Comprenons-nous bien: cette édition "scientifique", basée à la fois sur une grande érudition et sur une grande familiarité avec les poèmes de Nelligan, tient (dans l'appareil du tome I) un discours tel, typique de la critique d'identification, qu'elle seule obtiendrait la nelliganité du texte ${ }^{11}$, en .le dégageant autant que faire se peut des intermédiaires, y compris Dantin et Lacourcière. (I, p. 29), bien qu'elle ne puisse faire autrement, bien évidemment, que de devenir elle-même l'actuel intermédiaire: "Notre souci de respecter le plus scrupuleusement l'authenticité nelliganienne commande d'identifier 
toujours clairement ce que d'aucuns pourront appeler parfois nos ingérences * (I, p. 37). Pour le truchement, la question tient, en effet, dans le comment : limiter - de toute façon - le scandale en lui donnant forme esthétique ${ }^{12}$.

Comme si la nelliganité ne pouvait appartenir, en quelque sorte, qu'au tome I (qui couvre, il ne faut pas l'oublier, la totalité des années d'écriture: 1896-1941), comme s'il n'y avait pas dans le tome II, estime pourtant Michon, environ 35 cas - ceux qui sont signés et qui n'ont pas d'avant-textes connus ( 15 cas); enfin les textes sans signature et sans avant-texte (une vingtaine de cas) que nous appelons les "textes orphelins". (II, p. 25) - où il est possible que ce soit des textes d'une - réelle invention - (pour reprendre les mots de Robidoux et Wyczynski):

Sont-ce des textes de Nelligan? Le poète aurait-il cité de mémoire des textes demeurés jusqu'ici inédits? Aurait-il écrit tout simplement de nouveaux poèmes à l'hôpital? Ou sont-ce tout simplement des repiquages de poètes étrangers? À ces questions on ne peut répondre avec certitude. (II, p. 25)

Robidoux et Wyczynski, eux, ont déjà une certitude: "Chose sûre, il n'y a pas un texte, après 1899 , qui ne soit une tentative de recréation ou de mémoire à partir d'un original antécédent. (I, p. 28).

5. Petite difficulté technique exemplaire. Que dire, entre autres, du vers *irrégulier " suivant: "Oh! je veux être fou, ne fût-ce que" (I, p. 306)? Il est dans un sonnet d'“octosyllabes ", en effet, et pourtant le commentaire précise que ce vers particulier est *un vers de huit pieds composé de neuf mots " (I, p. 539)! D'une part, comment concilier syllabes (terme juste) et pieds (terme impropre)? D'autre part, bien que "fût-ce que " soit la dernière rime en "- usque- des quatrains, cela n'en fait pas une rime "normale", acceptable selon les canons de la métrique de l'époque: ne faut-il pas plutôt accepter qu'il y ait là un - vrai * vers faux ( 9 syllabes au lieu de 8$)^{13}$ et considérer que cette rime soit l'indice de ce faux lien qui reprend, métatextuellement, la folie du "je veux être fou ${ }^{14}$. Cela, bien sûr, n'est ni régulier dans le compte ni policé dans le traitement, au moins.

6. Le commentaire général, mais aussi les notes, dans le tome I, sont souvent plus qu'une annotation: presque une interprétation. Je pense ici aux nombreuses phrases mixtes (descriptives/allusives) du type

Les effets ainsi obtenus redoublent le poids des tristesses et des illusions fanées, incessante effusion lyrique dans un paysage automnal où les procédés formels, jusqu'au pluriel des mots, visent à créer une sorte d'ubiquité nostalgique. (I, p. 427).

Rien de tel dans le tome II, dont l'appareil est, si je puis dire, glacial. 
7. Enfin, les normes, sigles, abréviations et signes conventionnels, toujours dans le tome I, sont assez nombreux et plutôt lourds à manipuler, ce qui risque d'augmenter les erreurs et, autre paradoxe, les imprécisions. Ainsi, pour reprendre l'exemple cité plus haut, dans les variantes des "Corbeaux * (voir I, p. 273-274), il est impossible de comprendre que - mes vertèbres ", correction proposée par Dantin en 1909, est intégrée au texte, en fait, dès la seconde édition (1925): le losange plein placé après le sigle EP est utilisé * lorsque la leçon signalée réapparaît dans toutes les rééditions de l'édition princeps* (I, p. 51). Or, la leçon signalée est * nos ténèbres"!

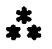

Le jeu tome I/tome II est désormais inévitable. Ce n'est pas parce qu'un ou plusieurs mots n'apparaissent pas dans les après-textes du tome II qu'ils n'ont pas été écrits dans les textes, voire dans les avanttextes du tome I (et surtout dans ceux dont les manuscrits n'ont pas été retrouvés ${ }^{15}$ ). Inversement, Nelligan a certainement décroché complètement avec le principe de rétroaction (des après-textes de l'internement sur les textes d'avant l'internement) pour inventer autre chose dans la réécriture ou pour écrire à nouveau: l'après-texte est probablement assez souvent un mixte de rétroaction (de retour à: reconstitution) et d'action (de retour sur: reconstruction $e t$ déconstruction). Le tome II ne peut pas être que le *révélateur * - comme en photographie - du tome I: il déborde, nécessairement. Le «texte de travail •(II, p. 9) qu'il est n'est en aucune manière ni un Émile Nelligan et son Guvre, tome II - à la manière, très pratiquée aujourd'hui, de Jaws, Jaus II -, ni un Poésies complètes 1896-1941, tome II. Et c'est plutôt, me semble-t-il, entre overground (Delahaye et Morin, Loranger et Saint-Denys Garneau, au moins) et underground (Nelligan) qu'il faut regarder.

Les CEuvres complètes et leur appareil critique (ample et riche) devraient permettre enfin d'y voir clair. La suprématie du tome I, qui couvre essentiellement le territoire des Poésies dites complètes de l'édition Lacourcière, n'est, pour ainsi dire, plus pensable, maintenant que le tome II est là: on peut les "confronter ", autre terme techniquè. Le diptyque, en effet, est désormais scriptible, au sens de Barthes.

1. Émile Nelligan, Euures complètes I. Poésies complètes 1896-1941, sous la direction de Réjean Robidoux et Paul Wyczynski, Montréal, Fides, 1991, 646 p.; CEuvres complètes II. Poèmes et textes d'asile 1900-1941, sous la direction de Jacques Michon, Montreal, Fides, 1991, 615 p. 
2. Emile Nelligan (1879-1941): cinquante ans après sa mont, colloque organisé par le Centre de recherche en civilisation canadienne-française (Yolande Grisé) de l'Université d'Ottawa, a eu lieu à la Bibliothèque nationale du Canada (18-20 novembre 1991).

3. Pierre H. Lemieux, Nelligan amoureux, Montreal, Fides, 1991; Gerald Godin, Nelligan revisité, Montréal, L'Hexagone, coll. .Lectures,, 1991; Aude Nantais et JeanJoseph Tremblay, Le Pontrait déchiré de Nelligan Montreal, L'Hexagone, coll. . Voies ", 1992. Le premier est un long essai, le deuxième un très bref essai (une -méditation. dit la quatrième de couverture), le troisième une -fiction dramatique. (adaptée du scénario, fait par les mêmes, de Nelligan, 1991, long métrage de Robert Favreau).

4. Tout ce qu'on connaît maintenant est, en quelque sorte, tout ce qui a été conservé. Pour ce qui est du tome I, les éditeurs écrivent: -Combien de pożmes au total? On ne peut le savoir. Plusieurs centaines peut-être, à réussite variable. On en a rescapé quelque 170 , tous conqus et crés en substance entre 1896 et l'été 1899 [...]. (I, p. 18). La même difficulté, ici, qu'avec les poèmes et proses de Rimbaud. Pour ce qui est du tome II, le gros morceau qui manque est la presque totalité (12 des 16 textes) du Quatrième Manuscrit (1935), actuellement bloqué par l'interdiction d'accès au Fonds Lacourcière (voir II, p. 255 et I, p. 12n), sans parler des textes transcrits sur feuilles détachées qui peuvent toujours surgir: voir les notes pour les textes FD.14, 15 et 16 (II, p. 540-543).

5. Si l'on inclut, pour le tome $I$, les - Miscellanea - (liste des - Pseudonymes, noms et patronymes., par exemple), a la limite de l'œeuvre et de son appareil critique, et si l'on exclut, pour le tome II, les quelques pages de présentation deś sept sections.

6. En 1989, à l'occasion d'une reproduction de cette édition (en petit format et amputée de ses - notes et variantes.), on en est rendu au $149^{\circ}$ mille (voir 1 , p. 572), ce qui fait de la section 1896-1899 de l'œuvre de Nelligan le *best-seller. incontesté de la poésie québécoise.

7. L'homme important dans tout ça: Paul Wyczynski, qui entre en scène nelliganienne, si je puis dire, tout de suite après la parution de l'édition Lacourcière, dès son arrivée au Canada. C'est essentiellement lui qui, depuis lors, a recueilli et diffuse ce que nous connaissons de Nelligan.

8. Je ne puis m'empêcher de joindre ce -mais. (et ce -néanmoins.) de l'avantpropos au -aussi - de la phrase qui le clôt et dans laquelle il est dit que l'édition de ces papiers - paraît aussi dans la collection "Le Vaisseau d'Or". Comme si cela avait pu paraître ailleurs! Voir note 14 .

9. Si je comprends bien ce que disent Robidoux et Wyczynski, :un exercice profondément créateur en devenir (en acte). (I, p. 25) est un exercice ou le travail est visible (.ratures, surcharges et repentirs.) et peut être constate comme tel, comme si la réécriture d'un texte déjà écrit (par soi ou par d'autres, peu importe) ne pouvait être considérée comme un exercice créateur: Robidoux et Wyczynski ne parlent-ils pas, pour le tome II, de -réécriture folle, incohérente et non maîtrisée. (I, p. 20). Qu'en est-il, alors, du travail effectué par Ducasse dans ses Poésies (1870) ou encore, dans le domaine plastique, par Duchamp dans ses ready-mades (à partir de 1913). Et Ponge qui, avec - Le verre d'eau. (1948, repris dans Méthodes, 1961), fait de l'ensemble de ses réecritures un seul texte? Et Borgès dont le . Pierre Ménard... Le Nelligan du tome II est a lire avec tous ces écrivains qui, sortant de la poésie comptée et rimée du XIX* siècle, ont mis en place le $\mathrm{XX}^{\bullet}$ siècle.

10. Comme me l'écrit Réjean Robidoux, le 22 novembre 1991, à la suite du colloque. En effet, nelliganien est l'adjectif qui revient le plus souvent dans l'avant-propos et l'introduction de ce tome.

11. A-t-on déjà entendu parler de la erimbaldité. du texte? Quelle édition est la plus - rimbaldienne:: celle de Jean-Luc Steinmetz (Paris, Plammarion, coll. .GP.), d'Alain Borer (Paris, Arléa) ou de Louis Forestier (Paris, Laffont, coll. - Bouquins .), toutes parues en prévision ou a l'occasion du centième anniversaire de sa mort 
(1991 également)? Voir là-dessus Jean-Jacques Lefrère: . Centenaire Rimbaud fin de l'épreuve, La Quinzaine litteraire, Paris, no 593, 16-31 janvier 1992, p. 7-10.

12. Michel Leiris, .De la littérature considéré comme une tauromachie (1945-1940), dans L'Âge d'bomme [1" édition: 1939], Paris, Gallimard, coll. .Folio, p. 14. J'implique ce texte relatif a la pratique de l'autobiographie, on l'aura compris, dans le contexte de la pratique de l'édition critique.

13. Quand on ne prononce pas le $e$ du .que. Autre exemple, cette fois d'heptasyllabes, tiré des :Loisirs de la poste: (1886-1898) dans les Vers de circonstance (1920) de Mallarmé: - Au charmeur des Muses becque- / té, plus prompt à l'estocade, / l'étincelant Henri Becque / rue, et 17, de l'Arcade. .

14. Voici qui, dès avant août 1899 , met en scène un décrochement téxtuel spécifique, dilatant le vers, semblable au décrochement qui, en 1929-1930, produira (13 syllabes au lieu de 12): :Hélas! il a sombré dans les racines du rêve!. (:Le Vaileau d'Or :, v. 14; II, p. 73). Comme l'écrit Tristan Corbière dans son -Epitaphe. (Les Amours jaunes, 1873): :- Ses vers faux furent ses seuls vrais.

15. Ceux du tome I dont les manuscrits ont été conservés ou retrouvés forment justement la matière des Poèmes autographes, presentation, classement et commentaires de Paul Wyczynski (Fides, 1991 toujours). Cet album semi-luxe reproduit donc la graphie manuscrite de 26 poèmes ou fragments de poèmes - "ce qui reste, en l'état actuel des recherches, de l'œuvre autographe du poète réalisée entre le printemps 1896 et l'été 1899. (p. 13) - ainsi que, sur la quatrième de couverture (juste en-dessous de - Collection "Le Vaisseau d'Or" ${ }^{n}$ ), un fragment du -Vaisseau d'Or - transcrit en 1937. S'ajoutent à cela une douzaine de photos, dont 5 du poète. Dans l'édition des CEuvres complètes, 11 textes autographes sont également reproduits ( 4 dans le tome I et 7 dans le tome ID). 